Economía, Sociedad y Territorio, vol. vIII, núm. 26, 2008, 357-379

\title{
Las políticas regionales compensatorias en el marco de la integración económica: reflexiones en torno al ALCA, los tLc y el Mercosur
}

\author{
JosÉ BRICEÑO-RUIZ \\ Dyanna María Ruíz-UzCátegui*
}

\begin{abstract}
The main premise of this paper is related to the fact that the Free Trade Area of the Americas (FTAA Or ALCA in Spanish), as an ambitious project of commercial openness, will have an enormous impact on the re-localisation of productive activities. This will therefore affect the less developed regions in the member countries. That is why, as it has been demonstrated by the experience in the European Union, it is necessary to establish compensatory policies through the creation of Regional Funds. These will allow some regions to alleviate the impact of the commercial aperture. Taking into account the way in which this issue had been addressed during the FTAA negotiations, this paper examines the convenience of establishing or not a free trading zone in the Western Hemisphere.
\end{abstract}

Keywords: economic integration, ALCA (FTAA), regional development, regional funds.

\section{Resumen}

El argumento central de este artículo es que el Área de Libre Comercio de las Américas (ALCA) — como ambicioso proyecto de apertura comercial- tendrá un enorme impacto en la relocalización de las actividades productivas, afectando a las regiones menos avanzadas al interior de los países miembros. Por esta razón, como lo demuestra la experiencia de la Unión Europea (UE), es necesario establecer políticas compensatorias mediante la creación de fondos regionales que permitan a ciertas regiones mitigar el impacto de la apertura comercial. Tomando en consideración la forma en que se ha tratado este tema en las negociaciones del ALCA, en este artículo se examina la conveniencia o no de instalar una zona de libre comercio en el hemisferio occidental.

Palabras clave: integración económica, ALCA, desarrollo regional, fondos regionales.

* Universidad de los Andes, Venezuela. Correos-e: bricenoruiz@hotmail.com, druiz03@yahoo.es. 


\section{Introducción ${ }^{1}$}

Una de las mayores críticas a la propuesta de establecer un Área de Libre Comercio de las Américas (ALCA) es el escaso tratamiento que se ha dado en las negociaciones a los aspectos sociales y las asimetrías que existen no sólo entre los países miembros, sino al interior de los mismos. Desde los noventa, estos temas se convirtieron en puntos relevantes en la nueva agenda de integración regional. En el caso de Europa, esta preocupación se remonta a décadas anteriores, como lo confirma la existencia de fondos de compensación y una creciente participación de los actores sociales en el proceso de toma de decisiones. Este ensayo tiene como finalidad evaluar y analizar las experiencias y avances de las políticas regionales compensatorias en materia de integración y su posible aplicación en el proceso del ALCA.

\section{La política de desarrollo regional en el proceso de integración europea}

La política regional es uno de los elementos del modelo de integración europea que algunos describen como dirigista o intervencionista, pues concibe la integración como un proceso que trasciende lo comercial y promueve el desarrollo económico integral y, en especial, la industrialización (Briceño Ruiz, 2001). En el modelo intervencionista, la integración se considera como un proceso cuyo objetivo no es fusionar mercados sino economías a través de la unión progresiva de los espacios económicos nacionales en una nueva entidad económica y política, caracterizada por la solidaridad entre sus miembros (Marchal, 1970: 124). Este tipo de integración se puede emprender mediante la adopción de un conjunto de medidas que faciliten la producción en gran escala, que permitan diversificar la producción y promuevan el progreso técnico. De acuerdo con este enfoque, la integración no descansa sólo en los mecanismos de mercado sino que requiere una participación del Estado como director del proceso. Como señala Vacchino, la integración "puede valerse del mercado y sus automatismos, pero, también y principalmente,

\footnotetext{
${ }^{1}$ Este artículo es parte de una investigación mayor titulada "Los productores agrícolas de los Andes venezolanos frente al ALCA: apertura comercial, fondos de compensación y equidad". Este proyecto cuenta con el apoyo financiero del Consejo de Desarrollo Científico, Humanístico y Tecnológico de la Universidad de los Andes, Mérida, Venezuela (Código NUTA-H-197-04-09-A).
} 
debe valerse de las acciones deliberadas y de las instituciones" (1982: 166). La integración debe hacer que correspondan las políticas de mercado con las que dirigen los Estados y las instituciones "de modo que sea factible eliminar las discriminaciones nacionales y originar un mercado unificado que, entre otras cosas, proporcione las directrices del proceso de desarrollo económico y social comunitario" (Vacchino, 1982: 162).

La integración europea se puede describir como intervencionista, ya que en ella se implementaron políticas de protección a los consumidores, de apoyo a las regiones menos favorecidas de los países miembros, inversiones en ciencia y tecnología, medidas de protección al medio ambiente, una política agrícola común, entre otros. Las ideas de Thomas Biersteker (1988) sobre el papel del Estado en la economía permiten validar el argumento sobre el carácter intervencionista del modelo de integración en Europa. Según este autor, es posible encontrar al menos seis modalidades de intervencionismo estatal en la economía:

- La primera modalidad se propone influir de manera positiva en la conducta de los empresarios a través de la política fiscal o la política monetaria.

- La segunda regula la conducta de los actores económicos privados o canaliza su actividad mediante políticas para proteger el medio ambiente, los programas de seguridad social o la legislación del trabajo.

- Una tercera modalidad de intervención busca transformar al Estado en mediador entre el capital y el trabajo.

- La cuarta es la redistribución del producto social y privado mediante subvenciones o por la política industrial.

- La quinta es la producción directa de bienes y de servicios.

- Finalmente, la modalidad de intervención del Estado en la economía es la planificación.

De acuerdo con las ideas de Biersteker, es fácil observar varios tipos de políticas intervencionistas en la integración europea. Por ejemplo, la legislación comunitaria de protección del medio ambiente o de defensa de los consumidores son, sin duda, modalidades de intervencionismo regulador. La política agrícola común es una forma de intervencionismo redistribuidor. El proyecto de construcción del Airbus es un ejemplo de intervencionismo cuyo objetivo es la producción de un bien en particular. 
En consecuencia, es válido describir la experiencia de integración en Europa Occidental como intervencionista.

La política regional, un componente de la integración europea desde sus inicios, es otra forma de intervencionismo redistribuidor. Desde fines de 1959 se adoptó un programa mínimo para tratar los problemas de las regiones con una sola industria o las regiones fronterizas. De igual manera, el Banco Europeo de Inversiones, creado en el Tratado de Roma, canalizó recursos para favorecer a las regiones menos desarrolladas y para financiar proyectos de interés común entre los países miembros (Pulgar, 1996). A inicios de la década de los setenta comenzó a configurarse de manera mucho más concreta una política regional cuando se creó en 1975 el Fondo Europeo de Desarrollo Regional (Feder), encargado de desarrollar, aplicar y financiar las políticas de desarrollo regional en la Comunidad Económica Europea (CEE). El Feder llevaría a cabo acciones para favorecer la realización de inversiones productivas, desarrollo de infraestructura, desarrollo endógeno, desarrollo local y de pequeñas y medianas empresas (Pymes), inversiones en educación y sanidad y proyectos piloto en las regiones fronterizas (González Vallve, 1993: 8).

Asimismo, el Acta Única Europea, suscrita en 1986, tuvo un efecto considerable en la política regional con la propuesta de crear un verdadero mercado único para 1982, meta alcanzada en la fecha prevista. A esto se sumó la propuesta del Tratado de Maastricht de establecer una unión económica y monetaria. Tanto el mercado único como la unión económica y monetaria tuvieron profundos efectos estructurales en las diferentes regiones de la UE. Por ello, en el Tratado de Maastricht se introduce la politica de cohesión que plantea, en lo fundamental, tres objetivos: la convergencia de los indicadores macroeconómicos, eliminar las disparidades del desarrollo regional y el desarrollo de la integración/interdependencia entre los diversos sistemas de producción regional y nacional.

Se observa que, desde sus inicios, la integración en Europa buscó proteger a las regiones más desfavorecidas económica y socialmente a través de programas de compensación que minimizaran las diferencias internas de renta y las oportunidades existentes entre las regiones de cada país miembro de la UE. Esta política regional de compensación estaba, y aún está, dirigida a redistribuir recursos y a promover la cohesión social, económica y territorial de la UE mediante transferencias de las regiones más desarrolladas a otras no tan avanzadas. 
Esta política destinada a reducir las disparidades regionales se basa en cuatro fondos estructurales: el Fondo Europeo de Desarrollo Regional (Feder), el Fondo Social Europeo (FSE), el Fondo Europeo de Orientación y Garantía Agrícola (FEOGA) y el Instrumento Financiero de Orientación de la Pesca (IFOP). ${ }^{2}$ En el periodo 2000-2006, con estos fondos se pagarán unos 213,000 millones de euros, lo que equivale a una tercera parte del gasto total de la UE. Asimismo, para este mismo periodo se asignó una cantidad adicional de 18,000 millones de euros para el fondo de cohesión. ${ }^{3}$

La asignación de estos fondos está sujeta a los siguientes criterios: $70 \%$ se destina a las denominadas regiones del objetivo 1 , cuyo PIB es inferior a 75\% de la media de la UE (entre esas regiones se encuentran, principalmente, Grecia, Portugal, España, sur de Italia y Alemania Oriental); $11.5 \%$ se asigna a las regiones de objetivo 2, aquéllas que se encuentran en situación de declive económico a causa de dificultades estructurales (por ejemplo, Dinamarca, país al que se asignaron 162 millones de euros para mejorar el transporte y las telecomunicaciones en las islas pequeñas y las comunidades costeras con acceso limitado por tierra y escasez de agua dulce). El objetivo 3 se centra en iniciativas y programas de creación de empleo en todas las regiones que no abarca el objetivo 1 . Otro $12.3 \%$ de los fondos se destina para adaptar y modernizar los sistemas educativos y de formación profesional y para otras iniciativas de fomento del empleo. Finalmente, 5.35\% se dedica a la cooperación transfronteriza, los programas de renovación urbana y el acceso justo al mercado de trabajo.

De acuerdo con el tercer informe sobre la cohesión económica y social de la Comisión Europea (2004), ${ }^{4}$ las diferencias de renta y de empleo tanto entre los países como entre las regiones de la $\mathrm{UE} 15^{5}$ se han reducido desde 1990, especialmente desde mediados de la década. De manera similar, el PIB per cápita en Grecia, España, Irlanda y Portugal fue muy superior a la media de la UE entre 1994 y 2001. En Irlanda, particularmente, en 2001

${ }^{2}$ Información disponible en http://europa.eu/pol/reg/overview_es.htm.

${ }^{3}$ El fondo de cohesión se creó en 1993 para financiar infraestructuras de transporte y medio ambiente en los Estados miembros que entonces tenían un producto interno bruto (PIB) inferior a 90\% de la media de la UE (Grecia, Irlanda, España y Portugal) y se ha ampliado a los nuevos Estados miembros. Debido a sus buenos resultados económicos, Irlanda dejó de ser beneficiada en 2004.

${ }^{4} \mathrm{http}$ ://ec.europa.eu/regional_policy/sources/docoffic/official/reports/cohesion3/ cohesion3_es.htm.

${ }^{5}$ Integrada por 15 países: Austria, Bélgica, Dinamarca, Finlandia, Francia, Alemania, Grecia, Irlanda, Italia, Luxemburgo, Países Bajos, Portugal, España, Suecia y Reino Unido. 
el PIB per cápita en unidades de poder adquisitivo era más de $17 \%$ superior a la media de UE15, mientras que a comienzos de 1990 era 25\% inferior a la media. El informe señala que el ejemplo irlandés demuestra de manera convincente la eficacia de la ayuda de los fondos estructurales cuando se combina con políticas nacionales orientadas hacia el crecimiento.

No obstante, la Europa de los 15 fue ampliada por la adhesión de los países del antiguo bloque soviético (República Checa, Hungría, Polonia y Eslovaquia), los tres Estados bálticos que una vez formaron parte de la Unión Soviética (Estonia, Letonia y Lituania), una de las repúblicas de la antigua Yugoslavia (Eslovenia) y dos países mediterráneos (Chipre y Malta). ${ }^{6}$

Con la adhesión de estos diez nuevos Estados, las diferencias de prosperidad tanto entre los países como entre las regiones se incrementó mucho más. Por esta razón, la Comisión Europea se propuso una política regional renovada y más integrada y una reforma a la política de cohesión de 2007-2013, cuyos principios en adelante serán: convergencia, competitividad y cooperación, para lo cual dispone de un presupuesto de 336,000 millones de euros (incluido el fondo de cohesión); lo que representa $35.7 \%$ del gasto total de la UE para ese periodo. Asimismo, la Comisión Europea dispuso dividir el gasto en tres partes: la primera, que representa $79 \%$ del total, se destinaría a reducir la brecha entre las regiones pobres y ricas; $17 \%$ se asignaría para aumentar la competitividad de las regiones pobres y para crear empleo local en las mismas; y el 4\% restante se centraría en la cooperación entre las regiones fronterizas. ${ }^{7}$

\section{2. ¿Qué se propone en materia de desarrollo regional en el ALCA?}

El ALCA es un proyecto de integración regional iniciado en la Cumbre de Jefes de Estado y de Gobierno de los países del Hemisferio Occidental, realizado en diciembre de 1994 en Miami, Estados Unidos. La propuesta original de establecer una zona de libre comercio desde Alaska hasta la Patagonia la anunció por primera vez en 1991 el entonces presidente de Estados Unidos George Bush, en su discurso sobre la Iniciativa para las Américas (IPA). Bush presentó un proyecto de integración que incluía la creación

\footnotetext{
${ }^{6}$ Para diez de estos países las negociaciones finalizaron el 13 de diciembre de 2002 en Copenhague, y se adhirieron en mayo de 2004, http://europa.eu/abc/12lessons/ index2_es.htm.

${ }_{7}$ Para más información, consultar http://europa.eu/pol/reg/overview_es.htm.
} 
de un espacio comercial único en el continente americano, la promoción de las inversiones y la condonación de la deuda que algunos países tenían con instituciones públicas estadounidenses. En diciembre de 1994, el presidente William Clinton decidió convocar a los presidentes y primeros ministros del continente en una reunión cumbre, en la que se relanzó la propuesta de una zona de libre comercio.

Una vez anunciado el proyecto del ALCA, comenzó un periodo de prenegociación con la participación de los respectivos ministros de comercio. Esta fase se inicia con la reunión celebrada en Denver, Estados Unidos, en 1995 y concluye con la realizada en San José, Costa Rica, en 1998. La etapa de negociaciones formales comenzó en abril de 1998 durante la Segunda Cumbre de las Américas en Santiago de Chile. Los grupos de negociación establecidos versan sobre los siguientes aspectos: comercio, servicios, propiedad intelectual, política de competencia, agricultura, antidumping, subsidios y derechos compensatorios y solución de controversias. El primer anteproyecto del tratado se presentó en la Tercera Cumbre de las Américas realizada en Québec, Canadá, en abril de 2001 y el segundo se dio a conocer en la Conferencia Ministerial de Quito, realizada en octubre de 2002. No obstante, en la Iv Cumbre de las Américas, en Mar del Plata (noviembre 2005), la negociación multilateral manifestó su evidente colapso; lo que significó un cambio de estrategia, por parte de Estados Unidos, hacia los tratados bilaterales de libre comercio (TLC) con países de la región: Chile, Colombia y Perú.

El ALCA se puede concebir como un nuevo modelo de integración en el que predomina la liberalización comercial y la flexibilización de las normas relacionadas con el comercio (inversiones, propiedad intelectual, compras gubernamentales, medio ambiente, etc.). Por otra parte, el ALCA rompe con la teoría tradicional de la integración pues se presenta como una zona de libre comercio, primera etapa de la integración económica, en la que se procedería sólo al desmontaje arancelario. Sin embargo, a diferencia de las tradicionales zonas de libre comercio, en el ALCA se combina la desgravación arancelaria con otras políticas, como la libre circulación de inversiones o la regulación de la propiedad intelectual, medidas más propias de etapas como el mercado común o la unión económica. A pesar de estos ambiciosos objetivos, en las negociaciones no se ha planteado la posibilidad de que el ALCA trascienda -una vez perfeccionada la liberalización comercial- la etapa de zona de libre comercio para convertirse 
en una unión aduanera o un mercado común. Esto último ha sido fuertemente rechazado por sindicatos estadounidenses que se oponen a la apertura del mercado laboral de su país debido a la enorme asimetría de salarios entre Estados Unidos y las demás naciones del continente (Briceño Ruiz, 2001).

Frente al predominio de los temas comerciales, en el ALCA se observa una ausencia de preocupación por los desequilibrios internos provocados por la apertura. Si se revisa el proceso negociador del ALCA, es notoria la exclusión de la cuestión regional. Este aspecto ni siquiera mereció la creación de un grupo de trabajo durante la etapa de prenegociación que concluyó en la reunión ministerial de San José (1998). Una vez iniciado el proceso negociador, esta materia tampoco se incluyo en la agenda. Una breve revisión de los borradores de acuerdo permite validar este argumento. Es notoria la ausencia de un grupo de negociación dedicado al tema de los efectos regionales del ALCA. Considerando que en el ALCA las asimetrías pueden ser incluso mayores que las existentes en el Tratado de Libre Comercio de América del Norte (TLCAN), la ausencia de una política regional puede convertir al libre comercio hemisférico en un factor generador de desequilibrios en las diversas economías nacionales.

Es apenas en la reunión ministerial de Quito cuando se decide suscribir un Programa de Cooperación Hemisférica ( $\mathrm{PCH}$ ) para tratar de alguna forma los aspectos no comerciales de la integración. No obstante, el PCH no es parte integrante del tratado constitutivo del ALCA, lo que puede generar dudas sobre su aplicación. La reciente Cumbre Extraordinaria de las Américas, celebrada en Monterrey en enero de 2004, es el reconocimiento de la necesidad del tema del costo de la apertura como parte importante del proceso de integración en el continente americano.

Por todo esto, el ALCA y los TLC (proceso paralelo) se pueden describir como un modelo comercialista que suscribe y profundiza las ideas de la teoría tradicional del comercio internacional (Briceño Ruiz, 2001). Considerando la integración como un segundo óptimo, el enfoque comercialista la concibe como "una experiencia local de 'desvalorización' de las fronteras, justificable en tanto que etapa transitoria que serviría para ir eliminando, escalonadamente, las tarifas arancelarias y otras restricciones a los intercambios comerciales" (Vacchino, 1982: 154). En tal sentido, la integración debería dirigirse esencialmente a abolir las barreras y los obstáculos que impiden el libre flujo de bienes y servicios entre las diversas economías nacionales. De esta 
manera, se crearía un espacio económico integrado en el cual la actuación de las leyes del mercado permitiría intensificar la interdependencia económica entre los países socios.

A diferencia de la experiencia europea, en las negociaciones del ALCA no existe referencia alguna a una estrategia de política regional. En primer lugar, en el TLCAN -que para Estados Unidos es el modelo de integración que debe fundamentar al ALCA- lo regional, incluso en su limitada modalidad de integración fronteriza, es casi inexistente. En segundo lugar, la revisión del proceso negociador del ALCA, iniciado en 1995 con la primera reunión ministerial de Denver, evidencia la ausencia de discusiones sobre la cuestión regional en su agenda de negociación.

En el TLCAN no existe una política regional, ni siquiera de integración fronteriza, a pesar que la frontera entre Estados Unidos y México se considera una región problemática, incluso descrita por el Instituto de Integración Latinoamericana (Intal) como una región plan, cuya homogeneidad está dada por un objetivo o problema común a resolver. Aun con esto, antes de la firma del TLCAN fue notoria la ausencia de mecanismos binacionales de integración que tratasen de forma global la problemática fronteriza (Valenciano 1992: 33-35). Tan solo, y en gran parte debido a la presión de grupos de la sociedad civil estadounidense, en los acuerdos paralelos en materia ambiental y laboral se crearon dos comisiones, la Comisión Ambiental y la Comisión Laboral, además de un Banco de Desarrollo de América del Norte para financiar obras de infraestructura en la región fronteriza. Estas comisiones se encargarían de resolver los problemas en sus áreas de competencia con un marcado sesgo fronterizo. Sin embargo, se objeta que apenas tratan una parte de la problemática fronteriza entre Estados Unidos y México.

La cuestión regional en el TLCAN no se limita sólo a las áreas de fronteras, sino a la totalidad del territorio mexicano. A diferencia de la UE y de las iniciativas de integración en América Latina, en el TLCAN participan dos países desarrollados y uno en desarrollo, lo que de por sí es una asimetría. Esta asimetría es mucho mayor si se considera que uno de los socios desarrollados es Estados Unidos, primera potencia industrial del mundo, que es justamente la que comparte frontera con el socio subdesarrollado. Los efectos de tales asimetrías serán importantes en cuanto a la localización de la actividad económica en México y la distribución de la riqueza entre las diversas regiones mexicanas. 
John Saxe Fernández (1996) considera que el TLCAN generará un proceso de competencia entre las regiones de los tres países para atraer inversiones, lo que describe como un proceso de microrregionalización. Este autor divide a México en tres áreas: norte, centro y centro-sur. La primera se ubica en la frontera con Estados Unidos y es receptora de ingentes inversiones en la industria maquiladora proveniente de este país. Esta zona tiene enormes ventajas en comparación con las regiones centro y centro sur. La competitividad del México norte es mucho mayor que sus pares porque ya está expuesta a la globalización y porque disfruta de la cercanía con el socio mayor. En consecuencia, esta región es más atractiva para los inversionistas, que preferirán localizar allí sus inversiones.

La experiencia del TLCAN confirma la validez de este argumento pues desde que entró en vigencia se produjo un desplazamiento importante de inversiones hacia los estados fronterizos con Estados Unidos. Aunque las entidades receptoras de inversión puedan beneficiarse, es preciso evaluar los costos laborales y ambientales teniendo en cuenta que con la cercanía al mercado estadounidense, los inversionistas decidan establecerse para aprovechar normas ambientales menos rigurosas y más fáciles de evadir o buscar mano de obra barata. Además, si la región cercana a Estados Unidos se convierte en la más atractiva para los inversionistas, la relocalización de la actividad económica que se produzca ira acompañada de un nuevo patrón de inmigración doméstica e internacional que puede producir trastornos económicos y sociales (Duquette, 2002: 82). Así, si la inversión se concentra en estados como Baja California, Sonora, Chihuahua o Coahuila, las entidades pobres del sur, como Chiapas, Oaxaca, Quintana Roo, etc., se verán afectadas y su población buscará migrar al norte en busca de nuevos empleos.

En relación con este punto y de acuerdo con la información presentada por el Instituto Nacional de Estadística, Geografía e Informática (INEGI) acerca de las regiones socioeconómicas de México ${ }^{8}$ ubica en el nivel de bienestar más alto (7) al Distrito Federal con $8.83 \%$ de la población del país; en el estrato siguien-

\footnotetext{
${ }^{8}$ Elaboradas con información del XII Censo General de Población y Vivienda de 2000; para lo cual se utilizaron 15 indicadores relacionados con el bienestar de la población: infraestructura, calidad y equipamiento en la vivienda; salud; hacinamiento; educación y empleo, entre otros. El estrato número 7 representa la situación relativa más favorable; contrario al nivel 1, que es el menos favorable. Esta información se encuentra disponible en http://jweb.inegi.gob.mx/niveles/jsp/index.jsp? me = na\&ly= $00 \& l a=00 \& a t=0 \& n e=n a \& n t=50$.
} 
te (6) se encuentran: Aguascalientes, Coahuila, Jalisco y Nuevo León con 13.74\% de los habitantes; en el nivel 5 están Baja California, Baja California Sur, Chihuahua, Sonora y Tamaulipas, que concentran $11.22 \%$ de la población. El 23.17\% de los habitantes de México se ubican en el estrato intermedio (4) compuesto por: Colima, Estado de México, Morelos, Nayarit, Querétaro, Quintana Roo, Sinaloa y Yucatán. En el nivel 3 se encuentra $12.73 \%$ de la población y lo integran: Durango, Guanajuato, Michoacán, Tlaxcala y Zacatecas. En el nivel 2, compuesto por $19.6 \%$ de la población, se ubica: Campeche, Hidalgo, Puebla, San Luis Potosí, Tabasco y Veracruz. Finalmente, en el estrato de menos bienestar encontramos a Chiapas, Guerrero y Oaxaca; que integran $10.71 \%$ de la población total de México. Se observa que las regiones que se ubican en los dos últimos niveles de bienestar se encuentran al sur del país.

Al respecto, Mendoza y Villena (2006: 591) señalan que la dinámica económica se concentró en la región central del país integrada por el Estado de México y el Distrito Federal, en tanto que el norte y la zona del Golfo de México presentaron tasas de crecimiento moderadas. Sin embargo, a partir del acuerdo comercial la producción tomó fuerza en las entidades de la frontera norte debido a la cercanía con el principal socio comercial de México. Por su parte, las entidades del sur también se vieron afectadas con la entrada en vigor del tratado, toda vez que su crecimiento económico disminuyó de modo considerable. Esos efectos se debieron en gran medida a la localización geográfica así como a diversos aspectos institucionales propios de cada región.

Asimismo, estos autores indican que los resultados de la investigación realizada por Messmacher (citado en Mendoza y Villena, 2006), acerca del resultado de algunas reformas estructurales y del TLCAN, muestran que esos cambios tuvieron dos efectos en la economía mexicana. En el primero, de índole sectorial, la tasa de crecimiento del sector manufacturero se incrementó de manera considerable en la mayoría de las entidades a partir de 1993, de modo que fue uno de los más beneficiados después del sector de transporte y comunicaciones. Este crecimiento resultó más favorable en los estados donde se concentra la producción manufacturera, como los del norte. En el caso del segundo efecto, de carácter regional, la estructura productiva de los estados del norte permitió un mayor crecimiento durante el periodo 1993-1999. 


\section{Gráfica I}

Tasa de crecimiento promedio anual del PIB per cápita (\%)

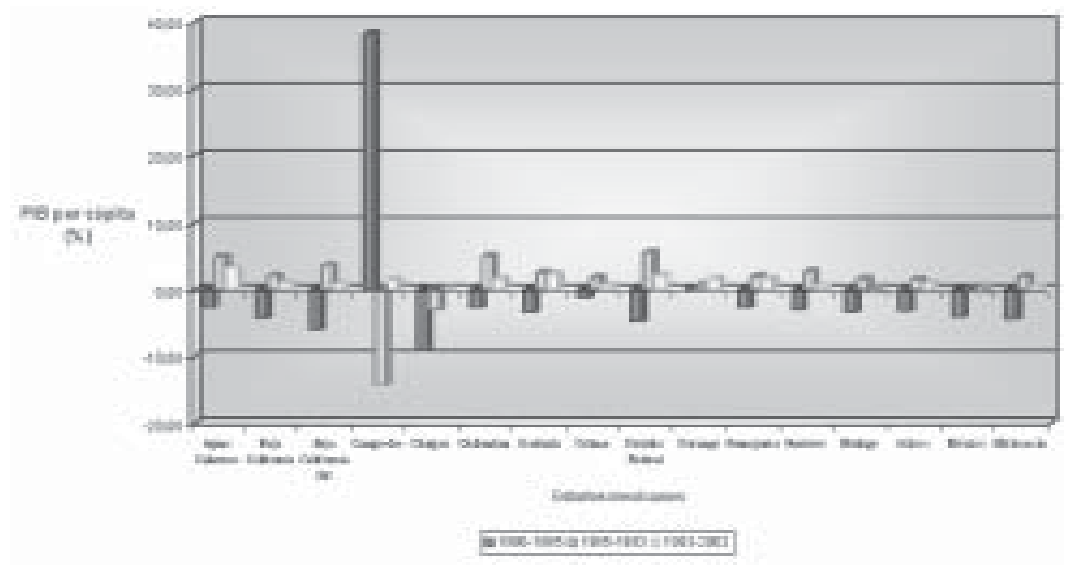

Gráfica II

Tasa de crecimiento promedio anual del PIB per cápita (\%)

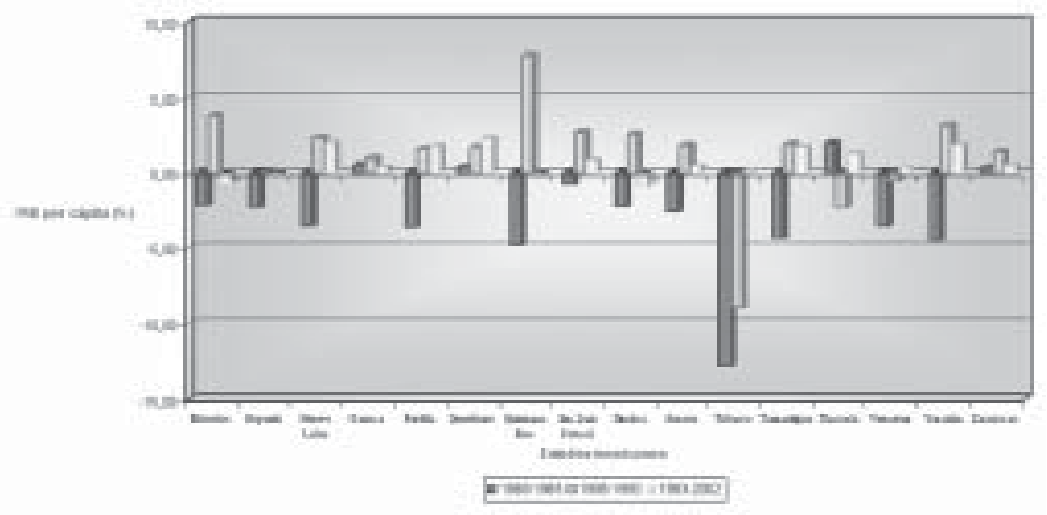

Fuente: Mendoza y Villena (2006), Comercio Exterior, 56(7), México, p. 585.

En relación con la gráfica I y II y de acuerdo con Mendoza y Villena (2006: 582), uno de los factores que explica la desigualdad económica regional en México es la dotación de infraestructura pública. Fuentes y Mendoza [citado en Mendoza y Villena, 2006] señalan que la asignación de dicha infraestructura desempeña un papel importante en las diferencias del PIB per cápita, ya que de 1980 a 1985 éste aumentó de manera significativa y apoyó un proceso de convergencia regional. En la etapa 1985-1993 esta varia- 
ble perdió importancia debido a la disminución de la participación del sector público en la provisión de bienes y servicios.

Asimismo, Díaz y Díaz (citado en Mendoza y Villena, 2006) indican que también incidió el nivel de escolaridad y la ineficiente formación de capital humano. Otra conclusión importante de Díaz (citado en Mendoza y Villena, 2006), es que los estados de la frontera norte son los más beneficiados por la integración económica de México, ya que sus niveles de PIB per cápita se elevaron de manera considerable como consecuencia de una mayor productividad manufacturera y el mejoramiento del capital humano de esa zona. Esto ocasionó que Baja California, Coahuila y Chihuahua incrementaran sus exportaciones a montos muy altos, además de que se cuentan entre los estados más beneficiados por la recepción de flujos de inversión extranjera directa que se tradujeron en una expansión de la industria manufacturera.

No obstante, como señala Rafael Velásquez (s/f.), el TLCAN no es el causante histórico de la disparidad entre el norte y el sur de México. El acuerdo simplemente vino a reafirmar y a acelerar una tendencia que se originó mucho antes. Asimismo, tampoco es el culpable directo de la crisis en la que se encuentra el sector rural del sur-sureste de México, ya que el abandono del campo mexicano tuvo su origen antes de la firma de dicho tratado. La falta de inversión pública del gobierno federal y la carencia de una política efectiva para el desarrollo regional son los factores que han incidido en la crisis del sector rural del sur-sureste.

Sin embargo, como señala este mismo autor, el TLCAN no ha disminuido la pobreza, la marginación ni el sector informal de la economía del sector rural del sur-sureste mexicano. En cambio, ha aumentado la migración de campesinos a los sectores urbanos y a Estados Unidos, ha ampliado la brecha educativa y tecnológica entre el norte y el sur de México y también ha profundizado la desigualdad en la distribución de la riqueza.

Al respecto, José Luis Calva (2005) señala que estas disparidades se mantienen y se profundizan por la ausencia de una política de desarrollo regional, ya que la integración de México al TLCAN en lugar de contribuir a reducir las desigualdades, más bien tendió a ahondarlas, ${ }^{9}$ debido a que priorizó áreas con ventajas

${ }^{9}$ De acuerdo con la Secretaría de Desarrollo Social de México (Sedesol), los estados con mayor grado de marginación son: Chiapas en primer lugar, Guerrero en el segundo y Oaxaca en el tercero; con menor marginación están Coahuila (29), Baja California (30), Nuevo León (31) y el Distrito Federal (32). Asimismo, el índice de desarrollo humano (IDH) para 2000 señala que el Distrito Federal se encuentra en el 
competitivas y dejó en el abandono a las regiones menos favorecidas, lo que ha ampliado la brecha entre regiones marginales y prósperas. Este mismo autor indica que en el TLCAN no hay un solo dólar para fondos compensatorios que palien los costos regionales de la integración. De forma similar, en México no existe una política integral de desarrollo regional para reducir progresivamente las desigualdades acumuladas.

Es decir, se plantea entonces lo que Clark Reynolds (1998) describe como el problema práctico de reconciliar a los ganadores y perdedores en el proceso de apertura. Así, surge la necesidad de cooperación no sólo entre los estados, sino entre las regiones involucradas para crear mecanismos dentro del TLCAN -responsable en gran medida de la aparición de estos problemas- que permitan diseñar una estrategia de desarrollo armónico en América del Norte. Reynolds (1998) también señala que, de hecho, ya se están formando coaliciones, por ejemplo, entre Cascadia y el corredor del sureste de Los Ángeles a Ensenada, esfuerzos iniciales de cooperación entre Arizona y Sonora o entre Texas y noroeste de México.

Por tanto, el modelo de integración del TLCAN ha excluido una política regional. Para Estados Unidos, el ALCA debe construirse según el modelo TLCAN, como lo demuestran sus propuestas que se centran en la apertura comercial, la promoción de inversiones y la desregulación de las áreas vinculadas con el comercio, sin incluir ningún tipo de medidas compensatorias, incluso entre países con tan dispares niveles de desarrollo como los que participan en la negociación del ALCA. En consecuencia, existen medidas compensatorias que merecen ser estudiadas, como las instauradas por la uE y las iniciativas de América del Sur en el marco del Mercosur y la Comunidad Andina de Naciones (CAN).

\section{La necesidad de una política regional a favor de la equidad en el marco del ALCA, los TLC y la iniciativa del Mercosur}

El ALCA y los TLC se proponen unir a países con enormes asimetrías. Sus efectos sobre las naciones y al interior de éstas serán devastadores si no se establecen medidas compensatorias a favor de los sectores y, en especial, de las regiones menos desarrolladas.

nivel más alto, seguido por Nuevo León y Baja California. Por el contrario, ocupan los últimos lugares: Guerrero, Oaxaca y Chiapas. http://seguimiento.sedesol.gob.mx/ documentos_especiales/index.php. 
La experiencia europea demuestra la necesidad de medidas redistributivas a través de una política regional, de manera que los menos aventajados no perciban la integración como un riesgo y, en consecuencia, se movilicen políticamente en contra de ella.

Todo proceso de integración tiene un efecto indiscutible en las economías regionales. La desgravación arancelaria y la mayor libertad de acción para los inversionistas producen una relocalización de las actividades productivas. Como el desarrollo económico es un proceso asimétrico, algunas regiones están más capacitadas que otras para soportar el impacto de la apertura, lo que profundiza los desequilibrios internos. Por ello, en la experiencia de integración en Europa, desde sus inicios se promovió una política regional basada en fondos de apoyo a las regiones más atrasadas.

Cada vez es más amplia la literatura que considera a las regiones nacionales como plataforma de la globalización y la integración económica (Boisier, 1993; Lisocka-Jaegermann, 1995). En varios países latinoamericanos esto ha conducido a un creciente interés por incluir la variable regional en los proyectos de integración económica, como es el caso de la Comunidad Andina que aprobó normas comunitarias como la Decisión 501 en la que se propone la creación de Zonas de Integración Fronteriza.

También en el Mercado Común del Sur (Mercosur) se ha intentado dar mayor importancia a las unidades políticas infranacionales, en particular a las ciudades, agrupadas en torno al Mercosur. Del mismo modo, en su Decisión 18/05 el Mercosur propuso en 2005 el Fondo para la Convergencia Estructural del Mercosur (Focem). Para llevar a cabo el Focem se desarrollaron los siguientes programas: 1) Programa de Convergencia Estructural que deberá contribuir al desarrollo y ajuste estructural de las economías menores y regiones menos desarrolladas, incluyendo el mejoramiento de los sistemas de integración fronteriza y los de comunicación en general. 2) Programa de Desarrollo de la Competitividad, cuya función es contribuir a la competitividad de las producciones del Mercosur -incluidos los procesos de reconversión productiva y laboral que faciliten la creación del comercio dentro de este organismo- y proyectos de integración de cadenas productivas y de fortalecimiento de la institucionalidad pública y privada en los aspectos vinculados a la calidad de la producción (estándares técnicos, certificación, evaluación de la conformidad, sanidad animal y vegetal, entre otros), así como a la investigación y desarrollo de nuevos productos. 3) Programa 
de Cohesión Social que contribuya al desarrollo social, en particular en las zonas de frontera, donde se podrán incluir proyectos de interés comunitario en áreas de salud humana y reducción de la pobreza y el desempleo. 4) Programa de Fortalecimiento de la Estructura Institucional y del Proceso de Integración que atienda la mejora de la estructura institucional del Mercosur y su eventual desarrollo.

Una vez cumplidos los objetivos de los proyectos, las estructuras y actividades que resulten serían financiadas por los países miembros, en función de la media histórica del PIB del Mercosur. Asimismo, las aportaciones de los Estados miembros del Focem tendrán el carácter de contribuciones no reembolsables y será de cien millones de dólares anuales; suma conformada de la siguiente manera: Argentina otorgará 27\%; Brasil 70\%; Paraguay $1 \%$ y Uruguay 2\%. Estos recursos se destinarán para financiar los programas y se distribuirán entre los Estados miembros de acuerdo con los siguientes porcentajes: para proyectos presentados por Paraguay, 48\%; para Uruguay 32\%; para Argentina $10 \%$ y para Brasil también 10\%.

En el proyecto para crear un Área de Libre Comercio Sudamericana (ALCSA) se promovió la Iniciativa de Infraestructura Regional Sudamericana (IIRSA), cuyo propósito es desarrollar vías de comunicación que conecten a las diversas zonas de los países sudamericanos. También existen iniciativas como el Fondo de la Cuenca del Plata (Fonplata) o el Fondo de Desarrollo del Caribe de la Asociación de Estados del Caribe (AEC).

En el ALCA y los TLC no existe ninguna iniciativa de este tipo. La integración hemisférica tendrá, sin duda, un efecto en la localización de las actividades productivas. ¿Cómo serán afectados los productores latinoamericanos?, ¿cómo incidirá esto en el desarrollo armónico de las regiones mas atrasadas de América Latina?, ¿cuál será el impacto del ALCA y los TLC en la frontera? No pretendemos responder a tales interrogantes en este breve ensayo. Ellas son, no obstante, parte de un necesario proceso de reflexión que conduzca a una cabal evaluación de los costos de ingresar al ALCA y a los TLC. Para ello, es menester recordar que en el grupo de negociación sobre inversiones, en el de negociación sobre servicios y en el de negociación sobre compras gubernamentales del ALCA existe un debate sobre si las disciplinas que se aprueben serán obligatorias para los gobiernos subnacionales (Bouzas y Da Motta Veiga (2002). 
Durante el proceso negociador del ALCA se excluyó la variable regional, a pesar del indudable impacto que sobre la localización de las actividades productivas tendrá esta propuesta de apertura comercial. Es apenas en la reunión del Comité de Negociaciones Comerciales (CNC) del ALCA, celebrado en Puebla en junio de 2003, cuando los países del Mercosur asoman la idea de incluir el tema de los fondos de compensación en la negociación. Meses después, en la reunión del CNC celebrada en Miami en noviembre de 2003, el gobierno venezolano formalizó la propuesta de establecer "un trato especial y diferenciado no sólo hacia la totalidad de cada una de las economías, sino también hacia el interior de cada una de ellas, de manera tal que dicho trato pueda dirigirse a las regiones y sectores que más lo necesitan" (Ministerio de Producción y Comercio, 2003: 20). En la reunión del CNC realizada en Puebla en febrero de 2004, la delegación venezolana reiteró la necesidad de tratar los desequilibrios regionales producidos por la apertura comercial. En el documento presentado por el gobierno venezolano se señaló que:

Ese escenario de grandes disparidades entre los países y en el interior de muchos de ellos, plantea el mayor de los retos al ALCA y obliga a que la atención de dichas disparidades ocupe un lugar predominante en el debate en torno a las negociaciones ya que de no ser así el propio funcionamiento del ALCA podría empujar a una profundización todavía mayor de las diferencias que caracterizan al escenario previo al inicio del Acuerdo (Venezuela, documento FTAA.sme/w/82/Rev.117 de febrero de 2004).

El gobierno venezolano reconoce que el tema de las asimetrías se ha discutido desde 1998 en el Grupo Consultivo sobre las Economías más Pequeñas. También considera valioso el Programa de Cooperación Hemisférica, aprobado en la reunión ministerial de Quito de 2002. Sin embargo, argumenta que:

Dichas iniciativas resultan insuficientes para una adecuada atención del problema, empezando por el hecho de que hasta la fecha el tratamiento especial y diferenciado no se hace extensivo a las economías que sin ser de las "más pequeñas" se caracterizan por su escaso grado de desarrollo promedio y/o por existir en su interior regiones o sectores que requieren de apoyos especiales para poder enfrentar con éxito el libre comercio hemisférico que el ALCA traerá consigo (Venezuela, documento FTAA.sme/w/82/Rev.117 de febrero de 2004).

Por ello, el equipo negociador venezolano propuso que se establezca un Fondo para el Fomento de la Producción (FFP), aun- 
que en el documento en cuestión no se delimitó de manera precisa la naturaleza del mismo. En vez de ello, se señalaron una serie de criterios generales que se describen en el anexo 1 de dicho documento.

La necesidad de establecer políticas que favorezcan a las regiones menos avanzadas es uno de los factores que explica el éxito de la integración en Europa. Existen razones para pensar que en América Latina es incluso más necesario el establecimiento de políticas de este tipo, particularmente válidas para las regiones fronterizas.

El ALCA y los TLC pueden agravar la situación de las regiones de frontera. Por una parte, es posible que la apertura comercial y la flexibilización de las normas sobre inversiones favorezcan a aquellas regiones que a los ojos de los inversionistas sean más atractivas por su infraestructura o cercanía con los centros económicos del hemisferio, en especial Estados Unidos. Sin embargo, una mayor integración económica significaría, al mismo tiempo, la intensificación de las actividades de transporte con las consecuentes secuelas sobre la infraestructura. Estos dos simples argumentos obligan a reflexionar sobre la necesidad de crear fondos de compensación que permitan a las zonas fronterizas de América Latina soportar el impacto de la apertura comercial.

De igual manera, si se produce una apertura indiscriminada del sector agrícola, los productores del campo pueden ser desplazados del mercado doméstico. No olvidemos que aunque en el ALCA la cuestión agrícola se está negociando sólo de forma parcial, excluyendo el tema de apoyos a la producción, sí se producirá una desgravación arancelaria y eventualmente una reducción o eliminación de los subsidios a las exportaciones. Productores muy competitivos como Brasil, Argentina o Uruguay se beneficiarán de una eventual reducción de aranceles. Pero, ¿cómo pueden enfrentar los productores agrícolas latinoamericanos este reto? La experiencia europea demuestra la necesidad de apoyar a las regiones agrícolas, para lo cual se aprobó la política agrícola común y se estableció el Fondo Europeo de Orientación y de Garantía Agrícola (FEOGA). Más recientemente, los europeos argumentaron sobre el carácter multifuncional de la actividad agrícola, por lo que es necesario que se apoye. Al margen de la validez o no de este argumento, lo cierto es que las regiones agrícolas europeas recibieron un tratamiento especial en el proceso de integración. Esto no existe en el TLCAN, base del modelo ALCA. 
Las regiones industriales también se incluyeron en la política regional de la UE. Desde el Programa Mínimo de 1959, las llamadas zonas industriales en decadencia recibieron el apoyo de Bruselas a través de medidas dirigidas a favorecer la reconversión industrial. Contrariamente, en el ALCA, como ocurre en el TLCAN, no existe ningún tipo de medida que permita a las regiones industriales venezolanas enfrentar los retos de mayor competencia de manufacturas provenientes de Estados Unidos o Brasil.

Finalmente, un eventual efecto de mayor apertura es el incremento de las desigualdades y asimetrías que existen entre las regiones latinoamericanas. Esto hace pensar en la conveniencia de crear fondos de cohesión para financiar proyectos de infraestructura y mejorar los servicios públicos. Esto se está haciendo en la etapa actual de la integración europea posterior a Maastricht.

La experiencia de Europa brinda algunos ejemplos del tipo de apoyo que se puede dar mediante estos fondos regionales: subvenciones a los productores agrícolas, mejoras en carreteras y otras vías, promoción de nichos industriales, ayudas a los transportistas -normalmente afectados por estos procesos de mayor competencia-, inversiones en salud y educación, etc. El interés en el desarrollo de políticas regionales supone medidas como inversiones internas activas, apoyo a las industrias y empresas de exportación competitiva a escala internacional y medidas para estimular al empresariado a la innovación y el aprendizaje (Acosta Puertas, 2004: 128).

Ciertamente, el financiamiento de estos fondos no es tema de poca importancia. Existen, sin embargo, aspectos que se deben considerar. Uno es el apoyo de instancias de financiamiento internacional, como el Banco Interamericano de Desarrollo o el Banco Mundial. Otra alternativa es optar por el modelo europeo y crear un fondo regional en el que los países más ricos que participan en el proceso de integración colaboren en su creación y mantenimiento. Esta idea, con todo lo difícil que pueda parecer si se toman en cuenta los intereses de las naciones más desarrolladas, daría un nuevo rostro al ALCA y a los TLC, pues los convertiría en un mecanismo de integración solidaria, para utilizar el lenguaje de la escuela estructuralista francesa, en el que los países miembros sumarían sus esfuerzos para alcanzar mejoras para todos los grupos sociales y la promoción de la equidad. 


\section{Conclusión}

La propuesta de crear fondos regionales de compensación en el marco de los procesos de integración regional, y en concreto en el caso del ALCA y los TLC, es un tema que merece debatirse en los ámbitos gubernamental y de la sociedad civil en América Latina. La integración económica y la apertura comercial, al igual que cualquier actividad económica, no son fines en sí mismas; son medios para lograr la meta concreta de mejorar las condiciones de vida de los ciudadanos. En otras palabras, son instrumentos que pueden ser útiles en la promoción de la equidad y en la lucha contra la pobreza. Estos mecanismos compensatorios han demostrado su eficiencia para reducir las disparidades entre los países, y sus regiones, que conforman la Unión Europea. Existen razones para pensar que en un proceso como el ALCA y los TLC, que reúnen a economías tan dispares, es una necesidad mayor. Es conveniente, en consecuencia, continuar realizando estudios técnicos y examinando casos concretos de aplicación de mecanismos compensatorios que mitiguen el impacto de la integración en las regiones latinoamericanas. Éste es un debate que ha sido fundamental en Europa, y más allá de las dificultades políticas que puedan existir para su inclusión en el ALCA o lejos de que se piense que es parte de una estrategia en contra del ALCA, merece que lo consideren los especialistas y quienes toman las decisiones en materia de integración.

\section{Bibliografía}

Acosta Puertas, Jaime (2004), “La integración y el desarrollo de las regiones de los países de la Unión Europea y América Latina”, Nueva Sociedad, 189, Caracas, pp. 125-140.

Biersteker, Thomas (1988), "Reducing the Role of the State in the Economy: The Politics of Stabilization and Structural Adjustment", ponencia presentada en la Reunión Anual de la American Political Science Association, Washington, D.C., agosto.

Boisier, Sergio (1993), Posmodernismo territorial y globalización: regiones pivotales y regiones virtuales, Documento 93/19, Serie Ensayos LC/IP/G.73, 4 de noviembre, ILPES, Santiago. 
Bouzas, Roberto y Pedro da Motta Veiga (2002), Evolución reciente del Mercosur y de las negociaciones del ALCA, Buenos Aires, Serie Mercosur, 12, Observastorio de la globalización de la Universidad de Barcelona, disponible en línea: http://www.ub.es/obsglob/mercosur.html.

Briceño-Ruiz, José (2001), "El modelo TLCAN de integración y las negociaciones del ALCA", Problemas del Desarrollo, 32(126), Universidad Nacional Autónoma de México, México, pp. 35-58.

Calva, José Luis (2005), “Desigualdades regionales”, El Universal, en <http://www2.eluniversal.com.mx/pls/impreso/ web_editoriales_new.detalle_h?var $=32673>, 5$ de octubre $\overline{d e} 2006$.

Duquette, Michel (2002), "Del pensamiento mágico al realismo global. El TLCAN y las nuevas tendencias en el desarrollo regional norteamericano", en José Briceño Ruiz y Ana Marleny Bustamante (coords.), La integración latinoamericana: entre el regionalismo abierto y la globalización, Consejo de Publicaciones de la Universidad de los Andes, Grupo Cefi, Mérida, Venezuela, pp. 67-90.

González-Vallve, José Luis (1993), La política regional de la Unión Europea, integración fronteriza y política regional: marco general en la Unión Europea, Centro de Formación para la Integración Regional, Montevideo (Cefir).

Lisocka-Jaegermann, Bogumila (1995), "Región, regionalismo e identidad hoy", Revista Interamericana de Planificación, XXVIII(112), Sociedad Interamericana de Planificación, Quito, Ecuador, pp. 22-29.

Marchal, André (1970), Integración y regionalización de la economía europea, Seminarios y Ediciones, Madrid.

Mendoza-Cota, Jorge Eduardo y Mary Villeda Santana (2006), "Liberalización económica y crecimiento regional en México", Comercio Exterior, 56(7), Bancomext, México, pp. 581-591. 
Ministerio de Producción y Comercio (2003), La República Bolivariana de Venezuela ante el ALCA, MPC, Caracas.

Pulgar, Telasco (1996), "Experiencias y modalidades de desarrollo binacional fronterizo en América Latina”, Capítulo SELA, 46, en <http: lanic.utexas.edu/_sela/AA2K/ES/N46/ rcap4610.htm>, 12 de septiembre de 2002.

Reynolds, Clark (1998), “The Political Economy of Open Regionalism", en James W. Wilkie y Clint E. Smith (eds.), Integrating Cities and Regions: North America Faces Globalization, Universidad de Guadalajara-UCla, Program on México-Centro Lucas Alamán para el Crecimiento Económico, A.C., México, pp. 1-75.

Saxe-Fernández, John (1996), "Mexamérica o la dialéctica entre macro y microrregionalización”, Nueva Sociedad, 32(1), Caracas, pp. 47-67.

Vacchino, Juan Mario (1982), “Teorías, esquemas y experiencias de integración económica regional”, Mundo Nuevo, año 5, 15-16, Caracas, pp. 154-182.

Valenciano, Eugenio O. (1992), "El Acuerdo de Libre Comercio México Estados Unidos y sus repercusiones en la frontera”, Integración Latinoamericana, 17 (181-182), Intal, Buenos Aires, pp. 21-46.

Velásquez Flores, Rafael (s/f.), “El impacto del TLCAN en el desarrollo regional de México: una perspectiva comparada entre el norte y el sur-sureste, en http://www.congreso.gob. $\mathrm{pe} / \mathrm{cip} / \mathrm{tlc} / 4 \_\mathrm{DOSSIER}$ TEMATICO/4_4/4_4_1/4_4_1_1/ $05 . \mathrm{pdf} \#$ se arch $=\% \overline{2} 2 \mathrm{el} \% 20 \mathrm{impacto} \% \overline{2} 0 \mathrm{de} 1 \% \overline{2} 0$ TLCAN\%20en $\% 20$ el $\% 20$ desarrollo\%20regional $\%$ 20de\%20m\%C3\%A9xico\%22>, 3 de octubre de 2006.

Recibido: 30 de enero de 2006. Reenviado: 13 de noviembre de 2006. Aceptado: 15 de enero de 2007. 
José Briceño Ruiz. Es candidato a doctor en ciencia política del Instituto de Estudios Políticos de Aix en Provence (IEP), Francia. Realizó estudios de posgrado en ciencia política en el IEP Aix, Francia, y relaciones internacionales en la Universidad de Durham, Inglaterra. Es profesor-investigador de la Universidad de los Andes, Venezuela; investigador asociado del Centro de Investigaciones sobre América Latina y el Caribe (CREALC) del IEP Aix y del Centro de Estudios Latinoamericanos Rómulo Gallegos (CELARG) en Caracas; es investigador nivel II del Programa de Promoción del Investigador del Ministerio de Ciencia y Tecnología en Venezuela; ha sido coordinador académico del Centro de Estudios de Fronteras e Integración (CEFI) y coordinador del Grupo de Estudios sobre Regionalismo, Integración y Desarrollo, ambos de la Universidad de los Andes. Ha sido editor de Aldea Mundo, revista académica especializada en fronteras e integración. Tiene varios libros escritos o editados sobre el tema de la integración regional y sus contribuciones sobre estos temas han sido publicadas en revistas de América, África y Europa.

Diana Ruiz. Es economista egresada de la Universidad de los Andes. En la actualidad realiza una maestría en fronteras e integración en la misma institución. Es profesora del Centro de Estudios de Fronteras e Integración (CEFI) de la Universidad de los Andes e investigadora del Grupo de Estudios sobre Regionalismo, Integración y Desarrollo. Sus investigaciones han sido publicadas en revistas nacionales e internacionales. 